\title{
Grundsätzliche Probleme der Versorgung mit funktionellen Knieorthesen
}

\author{
J. Grifka, H. Jutka \\ Orhop. Univ.-Klinik, St. Josef-Hospital, Bochum (Direktor: Prof. Dr. J. Krämer)
}

\section{Zusammenfassung}

Die Indikation zu funktionellen Knieor. thesen wird vor allem bei Insuffizienz des vorderen Kreuzbandes und zur postoperativen Versorgung gestellt.

Anhand der Analyse biomechanischer Untersuchungen wird die Schwierigkeit aufgezeigt, den Bewegungsablauf im Kniegelenk im einzelnen zu erfassen und bei der Knieorthesenversorgung nachzuahmen. Anamnese und klinische Untersuchung von Sportlern, die bei vorderer Kreuzbandinsuffizienz mit funktionellen Knieorthesen versorgt sind (24 Lenox-Hill, 25 C.Ti., 38 Donjoy), weisen exemplarisch auf die Probleme des praktischen Orthesengebrauchs hin. Problematisch ist insbesondere die Verschiebung der Orthesengelenke und eine ungenügende Schutzfunktion gegen die ventrale Translation des Tibiakopfes. Dadurch kommt es trotz Verwendung einer Knieorthese in 68\% der Fälle $z u$ evidenten giving-way-Ereignissen.

Bei den überprüften Kniegelenksorthesen sind die praktischen Probleme unabhängig von der Art der Schienengelenke. Die ärztliche Kontrolle sollte sich auf ein druckfreies Anliegen der Orthese und eine möglichst geringe Distalisierung richten.

\section{Basic Problems in Treatment with Functional Knee-Braces}

An indication for functional knee-orthoses is given especially with ACL-insufficiency and after ACL-operations.

Analyses of biomechanical examinations demonstrate difficulties in realizing the joint movement of the knee in detail and imitating it with knee-orthoses. An anamnestic investigation and clinical examination of sportsmen and -women, using functional kneeorthoses due to ACL-insufficiency (24 Lenox-Hill, 25 C.Ti., 38 Donjoy), demonstrate problems in practical usage of these orthoses. Problems especially arise from dislocation of the orthoses' joints and a deficient protection against anterior tibial translation. Thus leading to evident giving-way events though using a knee brace in $68 \%$ of these cases.

In the tested knee-orthotic-devices problems in usage are independent of the kind of mechanical joints of the orthoses. The clinical examination should prove an application of the orthosis without pressure and only little distal dislocation.

\section{Einjeitung}

Bei indikationsbezogener Einteilung von Knieorthesen kann zwischen prophylaktischen, rehabilitativen und funktionellen Knieorthesen unterschieden werden (Knoche, 1987).

In der ursprünglichen Zielsetzung waren rebabilitative Orthesen, wie diese postoperativ eingesetzt werden, durch stabilisierende und bewegungseinschränkende Elemente gekennzeichnet. Mit dem Übergang zur funktionellen Nachbehandlung sind hierfür weniger ruhigstellende und stützende Versorgungen gefragt, sondern es sind lediglich endgradige Bewegungslimitierungen gewünscht. Somit hat sich die ursprüngliche Zielsetzung der rehabilitativen Orthesen hin zu funktionellen Gesichtspunkten gewandelt.

Z. Orthop. 132 (1994) 207-213

(c.) 1994 F. Enke Verlag Stuttgart
Die Nachteile einer prophylaktischen Knieorthesen-Versorgung - allen voran die erhöhte Verletzungsgefahr bei prophylaktischem Orthesengebrauch (Teitz, 1987) - haben dazu geführt, daß diese Indikation allgemein abgelehnt wird (Rovere et al., 1987; Bentzin et al., 1988).

Die Indikation zur funktionellen Knieorthesen-Versorgung wird heute grundsätzlich in zwei Versorgungsbereichen gestellt: Einerseits - konform mit dem Wandel des Nachbehandlungskonzeptes - in der postoperativen Versorgung, andererseits bei Insuffizienz des vorderen Kreuzbandes, wie dies nach Traumen mit Teilrupturen, Totalrupturen und bei postoperativer Restinstabilität vorliegen kann.

Für den praktischen Einsatz funktioneller Knieorthesen muß sowohl eine Funktionsprufung hinsichtlich medizinischer Gesichtspunkte erfolgen, als auch eine entsprechende Akzeptanz von seiten des Betroffenen vorliegen. Oft handelt es sich um jüngere Sportler, bei denen der Schieneneinsatz auch im Training emptoh- 
len wird. Zumeist muß die Orthese bei typischen Verletzungsmechanismen funktionellen Schutz bieten. Trageverhalten und Akzeptanz werden von einer Reihe subjektiver Faktoren mitbestimmt (Grifka, 1990).

Für die Orthesenversorgung gibt es sowohl hinsichtlich allgemeiner biomechanischer Grundlagen der Gelenkgeometrie und der Kinematik bei physiologischen wie pathologischen Kniegelenksverhältnissen als auch bezüglich der konkreten, individuellen Funktionsbeurteilung erhebliche Schwierigkeiten, die im einzelnen aufgezeigt werden sollen.

\section{Anatomische Grundbedingungen und biomechanische Analyseversuche}

In anatomischen Untersuchungen werden die geometrischen Unterschiede zwischen medialer und lateraler Kondyle sowie medialem und lateralen Tibiaplateau hinsichtlich Größe und Kontur im einzelnen beschrieben. Kennzeichen von allgemeiner Gültigkeit sind, daß die laterale Kondyle in a.p.-Richtung länger ist als die mediale und daß bei beiden der posteriore Anteil einen kleineren Krümmungswinkel besitzt als der anteriore. Im Gegensatz zur Konkavität der knöchernen Kontaktfläche des medialen Tibiaplateaus ist der laterale Plateauanteil konvex gewölbt (Kapandji, 1985). Diese unterschiedliche Formvorgabe prägt den Bewegungsablauf, der summarisch in einer Roll-Gleit-Bewegung zusammengefaßt wird (Müller, 1982).

Um Bewegungsablauf und Konstruktionsprinzip des Kniegelenkes in Deckung zu bringen und eine verallgemeinernde Schlußfolgerung für die $\mathrm{Ki}$ nematik des Kniegelenkes zu ziehen, können zunächst drei prinzipiell unterschiedliche Arbeitsmethoden gegenübergestellt werden:

- In einem sogenannten kinematischen Lösungsansatz registrierte Nietert (1975) den Bewegungsablauf, um davon auf das Konstruktionsprinzip zu schließen. Er fertigte von dem Bewegungsablauf kinematographische Aufnahmen an und verfolgte die Bewegung einzelner markierter Punkte, um anhand von GesetzmäBigkeiten im Bewegungsablauf die Auswirkungen der Gelenkform zu analysieren. Dabei konnte er die Ergebnisse von Weber und Weber (1836, zit. nach Nietert, 1975 ) und Bugnion (1892, zit. nach Fick, 1911) bezüg. lich der Krümmungsradien der Femurkondylen nachvollziehen, deren Anordnung auch als Evolute bezeichnet wird (Abb. 1). Unter Berücksichtigung von Bahnkurven bei Bewegung von Oberschenkel und Unterschenkel bestimmte er mit Projektion auf den medialen Kondylus bei verschiedenen Kniegelenken die jeweilige Drehachse und definierte in einem approximativen Verfahren in einer Kreisfläche der höchsten Punktedichte dieser Drehachsen den Kompromißdrehpunkt, den er mit $42,3 \pm 3,6 \mathrm{~mm}$ in horizontaler Richtung von der frontalen Femurkontur innerhalb der Kondylen und 19,7 $\pm 3 \mathrm{~mm}$ oberhalb des Gelenkspaltes angab. Dabei konstatierte er, daß ein solcher Kompromißdrehpunkt für eine Kniegelenksflexion von mehr als $110^{\circ}$ nicht festzulegen sei.

- In umgekehrtem Ansatz zog Menschik (1987) Schlußfolgerungen aus dem Konstruktionsprinzip des Knie-



Abb. 1 Krümmungsradien einer Femurkondyle in der Seitenansicht nach Bugnion Laus Fick, 1911), die die Evolute bitden

gelenkes. um das Erscheinungsbild der Bewegung als Konsequenz der anatomischen Strukturen zu definieren. Dabei ging er von den physikalischen Formgebungen der beteiligten Strukturen einschließlich der Bänder aus, betrachtete diese in ihrem Zusammenspiel in Ruhelage und beschrieb daraus den von ihm definierten „Zwangslauf" des Kniegelenkes, den er als reproduzierbaren Bewegungsablauf aufgrund der physikalischen Bedingungen klassifizierte. Nach sukzessiver Durchtrennung von Bandstrukturen, bei Belassen der Kreuzbänder, kam er zu der schon von Burmeister (1888; zitiert nach Menschik, 1987) zur Nachahmung der Kniegelenksmechanik entwickelten Vier-GelenkKette. Hierbei ordnet Menschik (1987) die beiden Kreuzbänder als starre Stäbe ein, die eine überschlagene Stabkonstruktion mit jeweils zwei Ankerpunkten an Oberschenkel und Unterschenkel bilden.

- Börner et al. (1988) formulierten einen rechnergestützten, kinematischen Lösungsansatz. Mit dem Ziel, die optimale Position der Anbindung der Kreuzbänder zu analysieren, verwendeten sie die Finite-Elemente-Methode. Mit einem hoch leistungsfähigen technischen Berechnungsverfahren, das nach dem Minimalereignis-Prinzip funktioniert, legten sie unter Berücksichtigung der Gelenkkontur die isometrisch günstigsten Anbindungspunkte der Kreuzbänder für eine isometrische Beanspruchung über einen weiten Winkelbereich fest. Wie Börner et al. (1988) feststellen, wird damit eine qualitativ schwierige Berechnungsaufgabe, die eine exakte Lösung nicht mehr erlaubt, in eine qualitativ einfachere, quantitativ aufwendige $\mathrm{Be}$ rechnung überführt, die eine numerische Lösung ermöglicht. Dabei müssen zwangsläufig eine Reihe von Einzelfaktoren eliminiert werden. Die Aufgabenstellung ist bereits à priori auf die Kreuzbandisometrie ausgerichtet.

Diesen drei Vorgehensweisen ist gemeinsam, daß sie ihre Analyse und Schlußfolgerung auf die seitliche Betrachtungsebene, also einen zweidimensionalen Bewegungsverlauf projezieren. Außerdem beschränken sie sich bei ihrer seitlichen Betrachtung der Kontur von Femurkondyle und Tibiaplateau auf deren Relativbewegung zueinander ohne Berücksichtigung anderer Einflüsse. Nimmt man lediglich in einer simplifizierten, idealisierten seitlichen Darstellung die Patella mit ins Kalkül des Bewegungsablaufes, so ergibt sich bereits ein Sechseck der biomechanischen Punktbeziehung, das bewegungsabhängig ist und bezüglich der Krafteinwirkung kaum zu kalkulierenden Verschiebungen unterliegt ( $N \ddot{a}$ gerl, 1990: Abb. 2). 


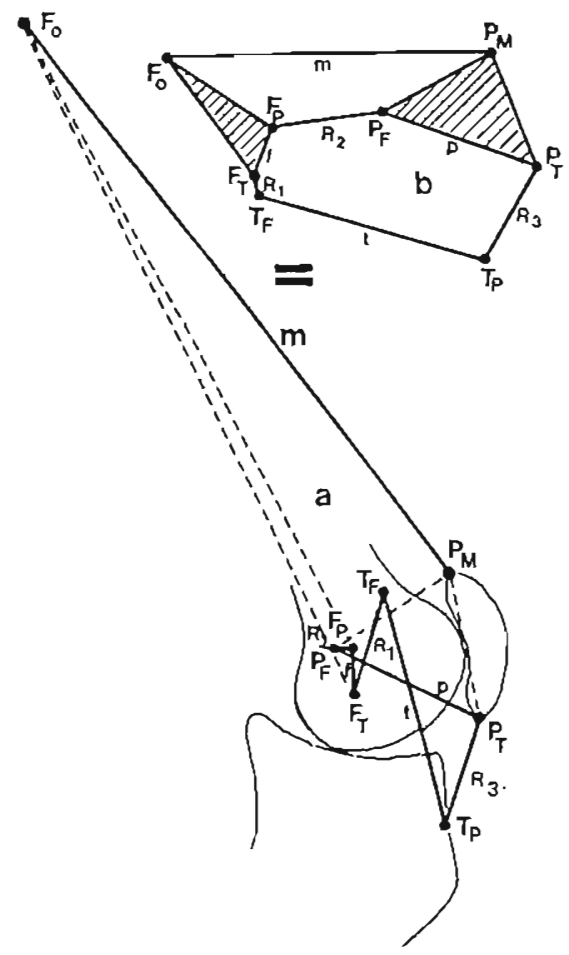

Abb. 2 Von Nägerl (1990) wird das Kniegelenk in planer, seitlicher Betrachtung von Tibia, Femur und Patella als geschlossene, sechsgliedrige kinematische Kette definiert, die neben Krümmungsmittelpunkten von der Patellaveränderung abhängig ist

\section{Raumkinematik}

Für die Analyse der Kniegelenksbewegung wird von Nietert (1975) und Menschik (1987) die Reduktion der dreidimensionalen Kinematik auf zwei Freiheitsgrade mit der sonst entstehenden Schwierigkeit der biomechanischen Berechnung und der während des Bewegungsablaufs lediglich endgradig angenommenen automatischen Schlußrotation begründet. Diese Annahme einer lediglich endgradig auftretenden automatischen Schlußrotation wurde von Schlepckow (1989) dahingehend revidiert. daß während des gesamten Beugungsbereiches eine Transversalrotation stattfindet.

Funktionsmäßig sind beim Kniegelenk jedoch 6 Freiheitsgrade zu berücksichtigen (Goodfellow und O'Connor, 1978). Dabei ist die Translation des Kniegelenkes (Noyes et al., 1989) nicht nur in der Ebene der Gelenkflächenkontakte, sondern in allen drei Bewegungsebenen $\mathrm{zu}$ konstatieren (Pennock und Clark, 1990). Pennock und Clark belegen mit ihren Studien auch, daß damit nicht nur drei Rotationsachsen $\mathrm{zu}$ berücksichtigen sind, sondern aufgrund der Mechanik der offenen Gelenkkette Bewegungszylinder resultieren. Dies bedeutet, daß das Kniegelenk nicht nur in allen Raumrichtungen Bewegungsmöglichkeiten hat, also Extension/Flexion, Abduktion/Adduktion, Innenrotation/ Außenrotation, medio-laterale Translation, antero-posteriore Translation und Distraktion/Kompression der Gelenkflächen, sondern durch die Kombination verschiedener Bewegungsanteile können aus jeder momentanen Stellung zahlreiche Variationen der Gelenkstellungsänderung eintreten.

Von maßgeblichem Einfluß sind schließlich die auf das Kniegelenk wirkenden externen Kräfte. Blankevoort et al. (1988) belegen. daß die tibiale Außenrotation bei Extension bei passiver Gelenkbewegung

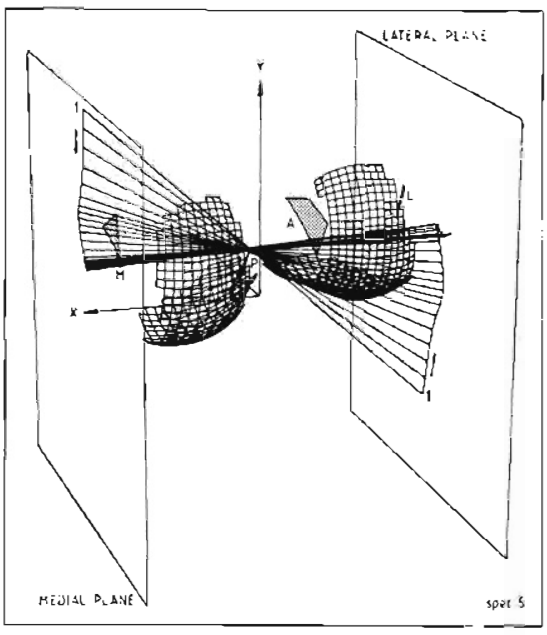

Abb. 3 Ansicht der Femurkondylen von posteromedial mit Darstellung der helixartigen Bewegungsachsen mit 1 als der Achse bei Beginn der Flexion und nachfolgender Achsverschiebung bei zunehmender Beugung laus Blankevoort et al., 1988)

nicht obligatorisch vorliegt, wohl aber als zwingendes Ergebnis bei achsialer Gewichtsbelastung festzustellen ist und dies zur zusätzlichen Stabilisierung beiträgt. Es versteht sich, daß die unterschiedlichen Krümmungsradien von medialer und lateraler Femurkondyle sowie die primären und sekundären Stabilisatoren für die während des Bewegungsablaufes kontinuierlich veränderte Bewegungsachse maßgeblich sind. Schlepckow (1989) konnte zeigen, daß das Rollen und Gleiten zeitgleich stattfindet und lediglich die Anteile beider in unterschiedlichem Verhältnis zueinander auftreten und $\mathrm{da} 3$ im lateralen Kompartment eine größere Gleitkomponente als im medialen vorliegt. Blankevoort et al. (1988) konnten nachweisen, daß sich die Bewegungsachsen bei passiver Durchbewegung zu dem Bild einer helixartigen Spindel summieren (Abb. 3).

Diese Betrachtungen zeigen, daß schon bei physiologischem Bewegungsablauf eine außerordentliche Komplexität vorliegt und selbst für den Individualfall nur bei Vorgabe einschränkender Randbedingungen Angaben des Bewegungsablaufes gemacht werden können. Eine Verallgemeinerung kann wegen der Vielzahl der Imponderabilien und bei Inkaufnahme erheblicher Schwankungsbreiten lediglich eine grobe Annäherung geben.

\section{Funktionelle Bedeutung des vorderen Kreuzbandes}

Bei unzureichender Funktion des vorderen Kreuzbandes, als häufigster Störung der Kniegelenksfunktion bei Kniebandverletzungen, kann es schließlich zur Desintegration des physiologischen Bewegungsablaufes, mit Instabilitätsgefühl und erhöhter Gefahr der Verletzung weiterer Kniegelenksstrukturen kommen. In Anlehnung an Jakob (1987) kann ein solches Knie als Risikoknie eingestuft werden.

Bei experimentellen Untersuchungen der passiven Durchbewegung des Kriegelenkes zeigt sich die größte Entspannung des vorderen Kreuzbandes zwischen $30^{\circ}$ und $60^{\circ}$-Beugung (Kiefer, 1989). Die Einwirkung äußerer Kräfte führt bei etwa $60^{\circ}$ in allen Anteilen des vorderes Kreuzbandes zu überproportionaler Längs- 
dehnungen (Kurosawa et al., 1991). Neben der Sicherung einer Ventralverschiebung des Tibiakopfes ist das vordere Kreuzband sekundärer Stabilisator gegen eine vermehrte Innenrotation (Daniel, 1990) und sorgt damit für die Transversalrotation mit der AuBenrotationsstellung bei Extension, was einer Relativverschiebung des lateralen Tibiaplateaus nach dorsal eatspricbt (Marans et al., 1989).

Bei Insuftizienz des vorderen Kreuzbandes ist diesur Bewegungsmechanismus gestört. wodurch eine virmehrte Translation des Tihiakopfes mil Verschiebung nach ventral resultiert (Marans et al.. 1989). Der Bewegungsablauf des Kriegelenkes ist auch ohne zusätzliche Streßeinwirkung in dieser Weise verändert. Markolf et al. (1976) fanden bei experimentellen Banddissektionen, da $B$ die alleinige Durchtrennung des vorderen Kruzbandes zur größten Ventralverschiebung führte. Daniel (1990) fand nach vorderer Kreuzbandresektion bei $30-90^{\circ}$ Flexion eine Translationsvermehrung um durchschnittlich $6.7 \mathrm{~mm}$ (2,8-13 mm).

\section{Praktische Orthesenanwendung}

Seit 1986 werden in der orthopädischen Universitätsklinik Bochum bei engagierten Freizeit- und Leistungssportlern mit nicht operativ versorgter vorderer Kreuzbandläsion bzw. postoperativer Restinstabilität funktionelle Knieorthesen zur externen Stabilisierung verordnet. Als Voraussetzung für die Versorgung müssen entsprechende giving-way Ereignisse vorliegen, sowie die Bereitschaft, die Orthese zum Sport zu benutzen. Bei den Trägern von 25 C.Ti., 38 Donjoy (beide polyzentrische Gelenke) und 24 Lenox-Hill-Knieorthesen (monozentrisches Gelenk) wurde nebed einer eingehenden klinischen Untersuchung und Befragung eine Funktionsprüfung der Orthesenversorgung durchgeführt. Das Alter der Patienten lag zwischen 17 und 38 Jahren mit einem Durchschnittswert bei 25 Jabren.

In einer Belastungserprobung wurde die Orthesenfunktion deskriptiv graduiert. Für die Prüfung des Stabilitätsgewinns durcb die Orthese erfolgte cine sonographiscbe Messung zur metrischen Bestimmung des Translationsausmaßes mil einer spizielled Meßvorrichtung (Grifka et al.. 1993).

Die Schienenanlage zeigte vur allem bei C.Ti. und Lenox Hill deutlicbe Problempunkte. So finden sich, unabhängig von den unterschiedlichen Kondylenpolsterungen. bei 10 der 24 Lenox-Hill-Trägern und bei 12 der 25 C.Ti.-Trägern Druckstellen im Kondylenbereich. Bei der C.Ti. ist dies vor allem im Bereich des lateralen Kondylenpolsters festzustellen. Bei der Donjoy-Orthese findet sich bei 7 der 38 Schienen ein ver. mehrter Druck im Bereich des lateralen Kondylenpolsters. In geringerer Zahl finden sich bei C.Ti. und LenoxHill Beschwerden ventral über der Tibia. Weichteilprobleme finden sich vor allem bei den Lenox-Hill-Schienen (11 Patienten $=46 \%$ ), weniger bei den C.Ti.-Schienen ( 5 Patienten $=20 \%)$. Im Weichteilbereich liegen vor allem Einschnürungen in der Kniekehle vor, die zu Druckbeschwerden mit Hautrötung und entsprechender Bewegungsbehinderung führen. Korrelierend zur Einschnü-

\section{Beugeeinschränkung bei Orthesenbenutzung}

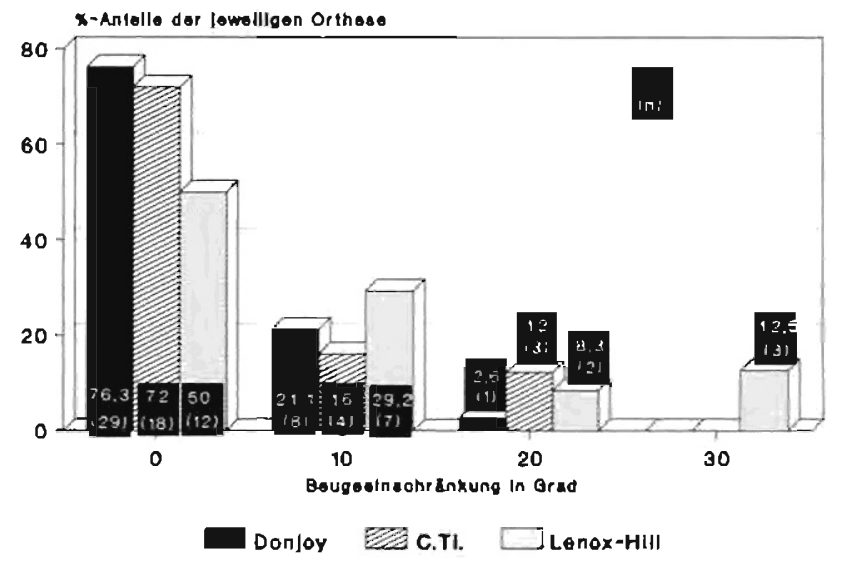

Abb. 4 Ausmaß der Beugebehinderung durch die verschiedenen Knieorthesen

rung der Bandzüge im Bereich der Kniekehle zeigt sich eine Beugebehinderung, die bis zu $30^{\circ}$ beträgt (Abb. 4). Bei der Donjoy-Schiene ist die Flexionseinschränkung vergleichsweise geringer. doch ist als besonderes Problem anzuführen. daß zwei Donjoy-Träger in der frühpostoperativen Phase eine tiefe Beinvenenthrombose entwickelten.

Bei maximaler Durchbewegung zeigen sämtliche Scbienen ein deutliches Auswandern der Gelenkachse. Dabei ist es gleichgültig, ob es sich um ein Einachsgelenk. wie bei der Lenox-Hill, oder um ein Doppelgelenk, wie bei C.Ti.- oder Donjoy-Schiene handelt. Sowohl in maximaler Extension als auch bei schon leicht vermehrter Flexion wandert das mechanische $\mathrm{Ge}$ lenk nach ventral (Abb. 5a, b). Dadurch verschiebt sich auch die ventrale Tibiakopfauflage der Orthesen nach vorne. so daß schon von daher die gewünschte Sicherung gegen anteriore Translation vermindert ist.

Bei maximaler Flexion treten bei starren Rahmenkonstruktionen weitere Hebelwirkungen und Orthesenverschiebungen auf. Bei sämllichen starren Rahmenkonstruktionen zeigt sich ein Abhebeln der kniegelenksfernen Orthesenanteile (Abb. 6). Bei den Donjoy-Orthesen mit weichen, nachgiebigen Ober- und Unterschenkelfassungen zeigt sich in $2 / 3$ der Fälle. daß die seitlichen Gelenkführungen nicht nur in a.p.-Richtung und nach distal auswandern. sondern bei maximaler Flexion ebenso seitlich auseinandergehebelt werden (Abb. 7).

Die Sportler tendieren dazu, die Schiene mit den Kondylenpolstern zu weit distal zu plazieren. zudem zeigt sich bei $2 / 3$ der Orthesen schon nach wenigen Beugebewegungen und kurzer Laufstrecke eine Distalisierung der gesamten Schiene, unabhängig vom Modell.

Für die Ermittlung des Stabilitätsgewinns durch die Orthese wurde eine sunographische Kontrolle der Relativbewegung zwischen Ober- und Unterschenkcl ohne Schiene und mit Anliegen der Schiene durchgeführt. Unter Berücksichtigung der Probleme der Schall- 




Abb. 5 Deutliches Auswandern der Orthesengelenkachse bei Beugung a) Ausgangssteliung in geringer Kniegelenksflexion

b) Auswandern der Schienengelenxe nach ventral bei schon mittelgradiger Flexion

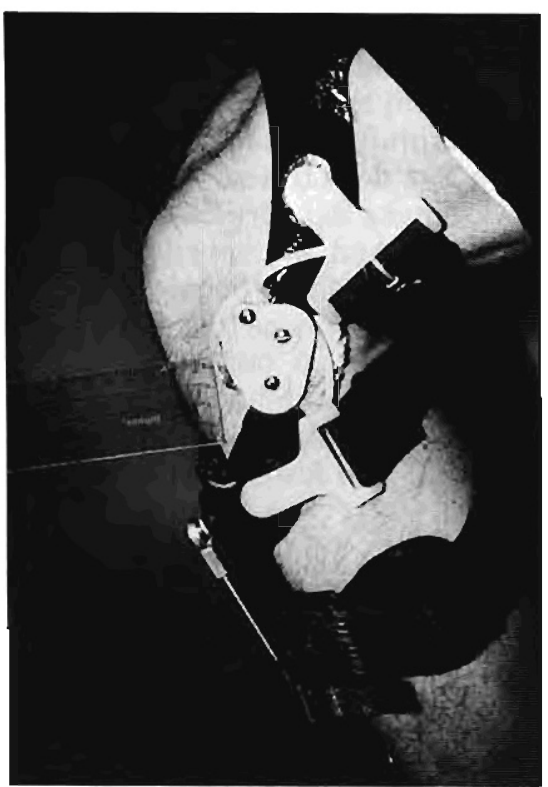

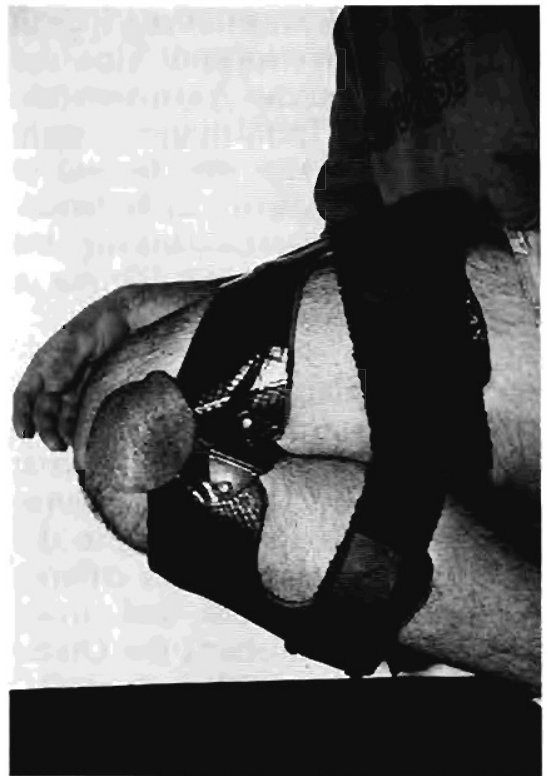

Abb. 6 Unzureichendes Anliegen der starren Rahmenkonstruktion bei Flexion. Oberschenkel- und Unterschenkelhalterung werden in inren ventralen Anteilen abgehebelt kopfanlage in der Kniekehle bei dort kreuzenden Gurtungen zeigt sich bei mehr als der Hälfte der C.Ti.$(56 \%)$ und Lenox-Hill-Schienen $(54 \%)$ keine wesentliche Reduktion des Instabilitätsausmaßes in anteriorer Auslenkungsrichtung. Bei der Donjoy-Schiene war in 30 Fällen $(79 \%)$ kein nennenswerter Stabilitätsgewinn in der sagittalen Ebene festzustellen.

Anamnestisch wurden von $59(68 \%)$ der 87 Patienten rezidivierende giving-way Ereignisse bei Orthesenverwendung angegeben, unabhängig von dem vorliegenden Instabilitätsausmaß.

Zu diesen objektiv erfaßbaren Schwierigkeiten und Mängeln der Schienenversorgung addiert sich die subjektive Einschätzung des versorgten Sportlers. Als Hauptgründe für die negative Einstellung der Schienenanwendung beim Sport werden vor allem ein umständliches Anlegen der Schiene, das Verrutschen der

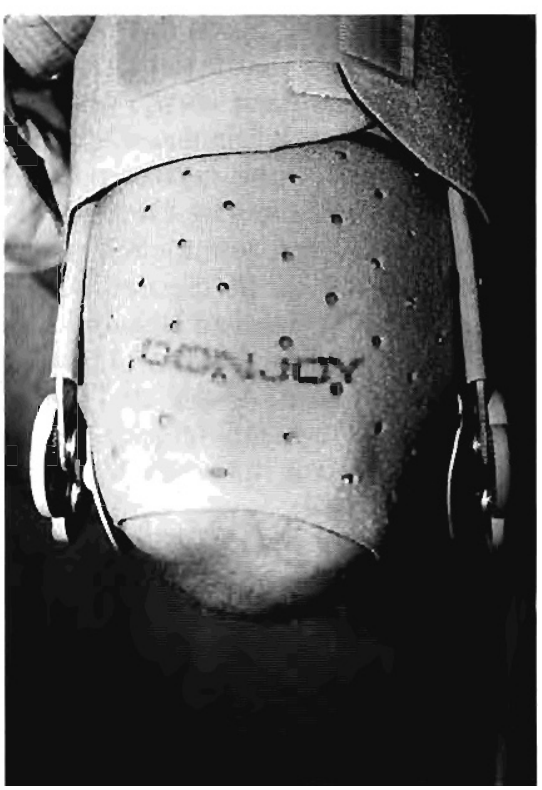

Abb. 7 Seitliches Auseinanderweichen der Gelenkführungen bei nachgiebigen Oberund Unterschenkelschienenfassungen

Orthese und die Entwicklung von Druckstellen, das Schienengewicht und schließlich die Bewegungseinschränkung genannt. Als Konsequenz dessen wird die Orthese nur von einem Teil der versorgten Patienten ständig zum Sport getragen $(11 \mathrm{C} . \mathrm{Ti}=44 \%: 7$ LenoxHill $=29 \% ; 22$ Donjoy $=58 \%$ ).

\section{Inkongruenz zwischen Kniekinematik und Orthesenmechanik}

Grundsätzliches Ziel der funktionellen Orthesenversorgung muß die externe Stabilisierung zun Ausgleich des vorliegenden Funktionsdefizites sein, um eine Schutzwirkung gegen evidente wie auch latente Instabilitäten zu entwickeln. Diese Hauptfunktion der funktionellen Orthesenversorgung ist von eminenter Bedeutung sowohl für die postoperative Versorgung als auch für die Schutzwirkung bei sportlicher Aktivität der 
oft jüngeren Erwachsenen. Die Idealvorstellung ist, mit einer funktionellen Knieorthesenversorgung eine der vorderen Kreuzbandfunktion äbnliche Verspannungswirkung zu entwickelo und somit Translationsbewegungen des Tibiakopfes entgegenzuwirken. Eine besondere Schwierigkeit liegt in der Ausgewogenheit der Stabilisierung ohne übermäßige Bewegungseinschränkung bei ausreichender Verhinderung der Instabilität (Grifka et al., 1990).

Mit dieser Zielsetzung wird die Hoffnung auf starre, steife Orthesenteile mit rückverlagerten monozentrischen oder polyzentrischen Gelenken gesetzt (Liggins und Bowker, 1991). Starre Materialien können allerdings bewegungsbedingten Konturänderungen der Muskulatur nicht nachkommen. Aufgrund des differierenden Bewegungsablaufes von Kniegelenk- und Orthesengelenk kommt es zu einer Verschiebung der Orthesengelenke, wie schon von Schrader (1929) festgestellt.

Durch dieses Auswandern der Orthesengelenke von der postulierten Kniegelenksachse büßt die Orthese ihre Führungseigenschaft bei Flexion ein (Regalbuto et al., 1989; Ulrich, 1992), und durch das ventrale Auswandern der Orthesengelenke wird der Translationsbewegung des Unterschenkels nach vorn Vorschub geleistet. Die Gefahr von Instabilitätsereignissen, die ohnehin typischerweise in Flexionsstellung auftreten, wird durch dieses Rutschen noch vergrößert. Die Ortheseneffizienz ist damit erheblich reduziert, womöglich sogar entscheidend aufgehoben, so daß es in diesem bohen Prozentsatz auch bei Tragen der Schiene zu Instabilitätsereignissen mit typischem giving-way kommt. Dies ist konkordant mit den Angaben von Millet und Drez (1988) sowie Rink et al. (1989), die rezidivierende Luxationen des Tibiakopfes nach ventral auch bei Verwendung üblicher Orthesen herausstellen. Somit wird die gewünschte Schutzfunktion gegen ventrale Tibiakopftranslationen trotz Schienenversorgung nicht erreicht (Jakob, 1987).

Die Donjoy-Schiene als relativ nachgiebige, flexible Orthese, die Ober- und Unterschenkel lediglich mit Gurtungen umfaßt, hat neben dem mangelnden Gegenhalt gegen ventrale Translation des Tibiakopfes auch keine genügende seitliche Führung, was sich im Abhebeln der Orthesengelenkanteile zeigt. Das Auftreten von Beinvenenthrombosen in zwei Fällen der Versorgung mit Donjoy-Schiene ist gut dadurch zu erklären, daß die Patienten die elastischen Bandgurtungen möglichst straff anziehen, mit der Absicht, damit eine größere Stabilität dieser weichen, nachgiebigen Orthese zu erreichen. Die signifikante Verringerung des BlutfluBes durch funktionelle Knieschienen wird durch Styf et al. (1992) beschrieben.

Bei den am Unterschenkel abgestützten Kniegelenksorthesen kann es schon nach wenigen Bewegungen zur Distalisierung kommen. Hierzu trägt die kovische Form des Oberschenkels bei, als auch das Eigengewicht der Kniegelenksorthese. Damit wird die gesamte Schiene in eine kinematisch ungünstige Position verschoben. Durch die Distalisierung übt die Orthese außerdem einen Streckeffekt auf das Kniegelenk aus (Bähler, 1988).
Somit ist wegen der mangelnden Übereinstimmung der Bewegung von Knie- und Orthesengelenk keine Schutzwirkung gegen anteriore Translationen zu erreichen; im Gegenteil kann die sogenannte funktionelle Orthesenversorgung durch verschiebungsbedingt auftretende, nicht kalkulierbare Krafteinwirkung eine Zwangsführung des Kniegelenkes verursachen, die sich für die gesamten Kapselbandstrukturen negativ auswirkt (Ulrich, 1992: Bähler, 1988). Bei dieser Unzulänglichkeit hilft auch der Versuch der radiologischen Justierung der Orthesengelenke (Blauth et al., 1990) nicht, da die Schiene bei Extensions- und Flexionsbewegung zwangsläufig eine nicht mit dem Kniegelenk kongruente Bewegung vollzieht. Die Anpassung unter Röntgenkontrolle ist daher für den praktischen Gebrauch irrelevant.

Von praktischer Bedeutung sind Funktionstests bei Orthesenanwendung, die an klinische Tests zur Erkennung von Kniebandinsuffizienzen angelehnt sind, wie beispielsweise "one leg long hop" oder Tests mit "Zick-Zack-Lauf" (Gauffin et al., 1990). Bähler (1981) empfiehlt zur Paßkontrolle einen Test aus Aufstehen und Sitzen, Treppesteigen und kurzer Wegstrecke auf unebenem Gelände. Zur schnellen Orientierung in der Praxis kann eine Testung mittels mehrmaliger Maximalbewegung des Kniegelenkes durchgeführt werden. Eine solche klinische Überprüfung stellt derzeit die beste Möglichkeit dar, den Sitz der Orthese zu kontrollieren, um hiermit die Akzeptanz der Orthese zu verbessern. Gleichwohl sollten sich Arzt wie Sportler der Tatsache bewußt sein, daß der intendierte Schutz gegen Instabilitätsereignisse in der Sagittalebene auch bei Orthesenversorgung nur unzureichend ist und lediglich eine Reduktion multidirektionaler Instabilitäten durch die Verbesserung der seitlichen Gelenkführung möglich ist, wie auch von Mishra et al. (1989) festgestellt wird.

\section{Schluffolgerungen}

Für die Versorgung mit funktionellen Kniegelenksorthesen zeigen sich grundsätzliche Probleme, die durch die spezielle Biomechanik des Kniegelenkes bedingt sind. Der komplexe Mechanismus der Kniegelenksbewegung kann durch mechanische Orthesengelenke nicht adäquat nachgeahmt werden. Entsprechend muß sich der Arzt mit den Folgen dieser mangelnden Inkongruenz befassen und das Augenmerk auf einen möglichst guten Sitz der Orthese richten. Als vordergründigste Maßnahme zur Verbesserung der Akzeptanz der Orthese durch den Sportler ist die Vermeidung von Druckstellen zu nennen. Dies kann durch entsprechende Ausarbeitungen, wie Polsterungen der Schiene, geschehen. Für die Kontrolle des Schienensitzes empfehlen sich ein Funktionstest in Anlehnung an Bähler (1981) sowie mehrmalige Maximalbewegungen des Kniegelenkes. Hierbei solite die Orthese nach Durchführung nicht merklich distalisiert sein.

Arzt wie Sportler sollten sich dessen bewußt sein, daß eine Orthesenversorgung mit stabiler seitlicher Führung zu einer Reduktion multidirektionaler Instabilitäten führen kann, in vielen Fällen jedoch bei Persistenz der ventralen Translation des Tibiakopfes giving-way-Ereignisse auftreten, so daß man sich bei Or- 
thesengebrauch nicht in trügerischer Sicherheit wägen sollte. Um Mängeln der Knieorthesenversorgung nachzugehen, sollte die ärztliche Kontrolle unmittelbar nach Auslieferung und nach Schienengebrauch über sechs Wochen erfolgen.

Um die grundsätzlichen Probleme der funktionellen Knieorthesenversorgung anzugehen, wird es noch erbeblicher Anstrengungen für die weitere technische Entwicklung bedürfen. Mit mechanischen Orthesengelenken ist aufgrund des komplexen Bewegungsablaufes des Kniegelenkes kein Lösungsansatz in Sicht.

\section{Literatur}

Bähler, A.: Die orthopădietechnische Versorgung des Knies bein Sportler. MOT 100 (1981) 32-37

Bähler. A.: Die biomechanischen Grundlagen der Orthesenversorgung des Knies. Orthop. Technik 40 (1988) 52-59

Benzin. J., M. Weiger, L. Gutjahr: Prophylaktische Stabilisierung des Kniegelenkes durch Bandage (Brace) beim Eishockeyspieler. Sinn oder Unsinn? Sportverletz. Sportschaden 2 (1988) 178-179

Blankevoort, L., R. Huiskes, A, DeLange: The envelope of passive knce joint motion. J. Biomechanics 21 (1988) 705-720

Blauth, W., H.-W. Ulrich, H. J. Hahne: Sinn und Unsinn von Knieorthesen. Unfallchirurg. 93 (1990) 221-227

Börner, M., H. Huber, C. Marheck: Die Kreuzbänder als wesentlicher Stevermechanismus der Kinematik des Kniegelenks. Z. Orthop. 126 (1988) 617-624

Daniel, D. M., W. Akeson, J. O'Conner: Knee-Ligaments - Structure. function, injury and repair. Raven Press, New York 1990

Fick, R.: Handbuch der Anatomie und Mechanik der Gelenke. 3. Teil: Spezielle Gelenk- und Muskelmechanik. Fischer, Jena 1911

Gauffin, H., G. Pestersson. Y. Teghter. H. Tropp: Function testing in patients with old rupture of the anterior cruciate ligament. Int. J. Sports Med. 11 (1990) 73-77

Goodfellow. J.. J. O'Conner: The mechanics of the knee and prothesis design. J. Bone Joint Surg. 60-B (1978) 358-369

Grifka. J., R. Krïner, A. Rosemhal, K. Bernsmann: Akzeptanz von Knieorthesen nach vorderer Kreuzbandruptur. Orthop. Praxis 26 (1990) 297-306

Grifku, J., K. Bernsimann, R. Hillen: Sonographische Stabilitätsơiagnostik des Kniegelenkes. p 143-158. Venbrocks R. Salis-Soglio G. (Hrsg.) Jahrbuch der Orthopädie. 1993

$J a k o b, R$. P.: Indikation, Behandlung und Evaluation bei chronischer vorderer Kreuzband-Instabilität. Orthopäde 16 (1987) 130-139

Kapandji, I. A.: Funktionelle Aratomie der Gelenke. Enke. Stutgart 1985

Kiefer, H.: Experimentelle Untersuchungen zur Biomechanik des Kniebandapparates. Habilitationsschrift. Ulm 1989

Knoche. W.: Knieorthesen - Schwerpunkte der postoperativen Kniebehandlung. MOT 107 (1987) 64-67
Kurosawa, H., K. I. Yamakoshi, K. Yasuda. T. Sasaki: Simultaneous measurement of changes in length of the cruciate ligaments during knee motion. Clin. Orthop. Rel. Res. 265 (1991) 233-240

Liggins, A. B., P. Bow'ker: A quantitative assessment of orthoses for stabilization of the anterior cruciate ligament deficient knee. Proc. Inst. Mech. Eng. H (1991) 81-87

Marans, H. J. R. W. Jackson, N. D. Glossop, M. C. Young MC: Anterior cruciate ligament insufficiency: A dymamic three-dimensional motion analysis. Am. J. Sports Med. 17 (1989) 325-332

Markolf, K. L., J.S. Mensch, H. C. Amsiuz: Stiffness and laxity of the knee - the contributions of the supporting struclures. J. Bone Joint Surg. 58-A (1976) 583-594

Menschik, A.: Biometrie - Das Konstruktionsprinzip des Kniegelenks. des Hüftgelenks. der Bcinlänge und der Körpergröße. Springer. Berlin 1987

Millet, C. W. D. J. Drez: Principles of bracing for the anterior cruciate ligament-deficient knee. Clin. Sports Med. 7 (1988) 827-833

Müller, W.: Das Knie - Form. Funktion und ligamentäre Wiederherstellungschirurgie. Springer, Berlin 1982

Nägerl, H.: Biomechanische Prinzipien in Diarthrosen und Synarthrosen. Habilitationsschrifł, Göttingen 1990

Nielert, M.: Untersuchungen zur Kinematik des meschlichen Kniegelenkes im Kinblick auf ihre Approximation in der Prolhetik. Dissertation, Berlin 1975

Noyes, F. R., E. S. Grood, P. A. Torzilli: The definitions of terms for motion and position of the knee and injuries of the ligaments. J. Bone Joint Surg. 71 - A (1984) 465-472

Pennock, G. R., K. J. Clark: An anatomy-based coordinate system for the description of the kinematic displacements in the human knee. J. Biomechanics 23 (1990) 1209-1218

Regalburo, M. A., J. S. Rovick, P. S. Walker: The forces in a knee brace as a function of hinge design and placement. Am. J. Sports Med. 17 (1989) $53.5-543$

Rink. P. C., R. A. Scon. R. L. Lupo. S. J. Guest: Team physician 7. A comparative study of functional bracing in the anterior cruciate deficient knee. Orthop. Rev. 18 (1989) 719-727

Rovere, G. D., H. A. Haupt. C. S. Yares: Prophylactic knee bracing in college football. Am. J. Sports Med. 15 (1987) II1-116

Schlepckow, P.: Experimentelle Untersuchungen zur Kinematik des stabilen und instabilen menschlichen Kniegelenkes. Z. Ortlsop. 127 (1989) 7) $1-715$

Schrader, E.: Über die Anweisungen der Verlagerung des technischen Knicschaniers zur physiologischen Gelenkachse. Z. Orthop. Chir. (1929) $451-463$

Styf, J. R., M. Nakhostine, D. H. Gershumi: Functional knee braces increase intramuscular pressures in the anterior compartment of the leg. Am. J. Sports Med. 20 (1992) 46-49

Teilz C. C.: Evaluation of the use of braces to prevent injury to the knee in collegiate football players. J. Bone J. Surg. 69-A (1987) 2

Ulrich, H.-W.; Knieorthesen - funktionclle Ansprüche aus der Siclit des Operateurs. Orthop. Technik 43 (1992) 174-182

Priv.Doz. Dr. med. J. Grifka

Orthop. Universitätsklinik

St. Jusef-Hospital

Gudrunstraße 56

D-44791 Bochum 